centrifugalisation with but little accompanying débris. 3. A second concentration method is described which does not necessitate the use of ether, and which is therefore more suitable for preparing cysts for cultivation experiments This consists of filtering the emulsion through silk, and subsequent fractional centrifugalisation. 4. The difficulties in the way of enumerating entamceba cysts are discussed, and the method found most suitable, by means of the coverslips used with Böttcher's slides, is described.

\section{A CASE OF SUPPURATIVE MENINGITIS WITH GLYCOSURIA SIMULATING DIABETIC COMA.}

BY FRANK E. TAYLOR, M.D., B.S. LOND.,

M.R.C.P. LoND., F.R.C.S. ENG., D.P.H. CAMB.,

PATHOLOGIST AND BACTERIOLOGIST TO THE QUEEN ALEXANDRA MULITARY HOSPITAL, MILLBANK ; LECTURER ON BACTERIOLOGY, UNIVIERSITY OF LONDON, KING'S COLLEGE AND

W. H. MoKInSTRY, M.B., C.M. GlasG., D.P.H. Lond., TEMPORARY CAPTAIN, R.A.M.C.

ON Oct. 13th, 1916, the following case was admitted into the Queen Alexandra Military Hospital, and was thought worthy of being placed on record on account of several interesting points to be discussed later.

Second Air Mechanic A-, of the R.F.C., aged 36, with four months' service, had been ill for 24 hours, complaining of severe pain in the right side of the chest, cough, and difficulty of breathing. On admission the patient appeared ill, with flushed face and marked dyspncea. The temperature was $102^{\circ} \mathrm{F}$., the pulse-rate was 112 , and the respirations were 38 per minute. Examination of the chest revealed diminished expansion of the right lower chest, with stony dullness and absence of tactile vocal fremitus and breath sounds of the right lower lobe. Typical "rusty" sputum was being expectorated, and the case was diagnosed as one of pleurisy and pneumonia. Poultices were applied to the chest. Calomel gr. iii. and pulv. ipecac. co. gr. $x$. were ordered statim.

Oct. 15th: The patient had had a bad night. Pain was intense, so that he could not lie comfortably in bed. The temperature still ranged from $101^{\circ}$ to $103^{\circ}$. The pulse was of good quality, with a rate of 100 . The respirations were very rapid, rising to 60 per minute. The physical signs in the chest remained the same, no signs of fluid being present. There was no cyanosis. The heart and pericardium were normal. The application of leeches to the right side of the chest caused great relief of the pain almost immediately, and a stimulant expectorant mixture was prescribed. Oct. 16th : Patient appeared to be greatly improved, said he felt quite well, and was very cheerful. The temperature was $102^{\circ}$, pulse 100 , and respirations 36 . There was very little cough and no expectoration.

Oct. 17th: The ward sister noticed some twitching of the patient's face; he had previously complained of intense headache. $\mathrm{He}$ vomited immediately afterwards and became very collapsed with incontinence of fæces. Coma rapidly supervened, with dilated pupils which did not react to light. Conjugate deviation of the eyes was noted first to the left and then to the right. All the superficial reflexes except the conjunctival were lost. Later he became very restless, constantly picking at his face, which wore a perpetual frown. The respirations rose to 64 and presented the appearance of air-hunger. The pulse still remained of good quality, being 100 to the minute. He had retention of urine, and a catheter was passed, drawing off $20 \mathrm{oz}$. On examination this contained a trace of albumin and a few hyaline and granular casts, as well as a large amount of sugar (not estimated quantitatively), but acetone and diacetic acid could not be detected. No distinctive odour of his breath could be recognised by any of several observers who examined him with this in view. Further, no history suggestive of glycosuria-excessive thirst, wasting, or polyuria - could be elicited on cross-examination of his wife; his health before this attack seemed to have been very good. An attempt made to administer alkalies subcutaneously was unsuccessful on account of the patient's restlessness, and rectal salines met with no better results. He could not take anything by the mouth. His condition remained much the same during the night.

Oct. 18th: The respirations were now 80 and the pulse 144 , and inclined to be irregular. His colour had much improved. The pupils were no longer dilated. He appeared to be temporarily paralysed on the left side, and had conjugate deviation of the eyes to the left. Incontinence of urine now

developed, but a sample of urine was collected and found to be quite free from sugar, whilst the quantity of urea present was normal. Venesection was performed, $20 \mathrm{oz}$ of blood being removed, and two pints of normal saline containing 1 per cent. sodæ bicarb. were injected into the vein. Later the eyes became deviated to the right, the pulse got weaker, Cheyne-Stokes respiration developed, the colour became cyanosed, spasms of the jaw and muscles of both arms were noted, and the patient died at 3P.M. Just before death the temperature rose to $107^{\circ}$. The tem. perature chart is here shown.

A post-mortem exa mination was made 36 hours after death. The body was well nourished and showed no signs of external injury except a hæmatoma about the size of the palm of the hand between the umbilicus and left anterior superior spine of the ilium. Thorax: The whole of the pleura covering the right lung was covered with a layer of semi - membranous purulent exudation about a quarter of an inch thick, which could easily be peeled off. The left lung and pleura appeared healthy. There was no free fluid in the pleural cavity. The heart appeared normal and there was no pericardial effusion. The vessels about the base of the heart and the structures

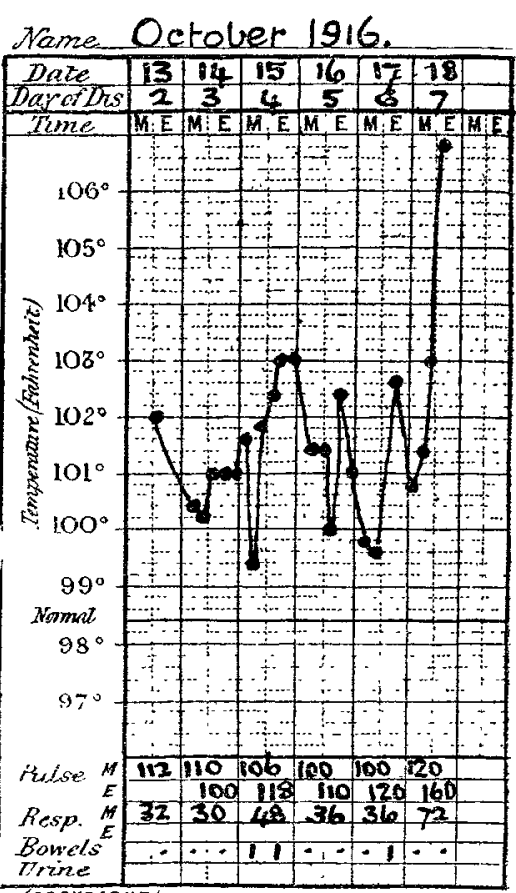

Temperature chart showing rise of in the mediastinum were

normal. Abdomen: All the organs, including the kidneys and liver and pancreas, appeared healthy. Brain: On removing the outer coverings of the brain pus was found mapping out the sulci on the anterior, superior, posterior, lateral, and internal surfaces, and also following the course of the blood-vessels. When the brain was removed the base was found free from pus except for a small area on each side where the middle meningeal artery takes its origin. The upper surface of the cerebellum was thickly covered with pus, particularly in the region adjoining the mid-cerebrum. The other surfaces of the cerebellum were free from pus. No pus could be found about the medulla. The brain when cut into showed no sign of abscess or excess of fluid in the ventricles.

Smears taken direct from the pus in the sulci, stained by Gram's method, showed long chains of streptococci as well as staphylococci. Streptococci were also found in smears from the cerebro-spinal fluid and in the blood drawn direct from the seared heart. Media of agar, broth, and trypsin legumin agar inoculated from the blood and the pus from the brain and pleura, incubated at $37^{\circ} \mathrm{C}$. for 24 hours and examined, showed growths of streptococci, Staphylococcus aureus, and a large Gram-positive sporing organism, the last being, no doubt, due to post-mortem invasion of the tissues.

Although the nature of this case was plainly revealed by the post-mortem examination, the recognition of the cause of the sudden coma formed a difficult and perplexing problem during life. It was in the attempt to settle this question that the urine was sent for examination, and when it was found that it contained a large quantity of sugar the diagnosis of diabetic coma was raised. The absence of polyuria and of acetone and diacetic acid in the urine, as well as the absence of the characteristic sweetish acetonelike odour of the breath, rendered this diagnosis a very questionable one.

The question of uræmic coma was next raised, seeing that albumin, though in small amount, as well as a few hyaline and granular casts were found in the urine. The determina tion of the percentage of urea was then suggested, and for this purpose a further specimen of urine was obtained on the following day. The percentage of urea was found to be normal (i.e., 2 per cent.), and this fact was taken as disposing of the diagnosis of uræmic coma. A quantitative determination of the sugar was then decided upon, but in this specimen no trace of sugar or other Fehling reducing substance could be obtained, showing that the glycosuria was 
only a transient condition. Lastly, the terminal hyperpyrexia, the temperature rising just before death to $107^{\circ} \mathrm{F}$., which occurs not uncommonly in infective conditions, is an interesting point to note.

As regards the relationship between diabetic coma and the presence of the acetone-bodies in the urine, Cammidge ${ }^{1}$ states that "all cases of diabetes are not complicated by acetonæmia, although it is a constantly present menace, since a deficiency in the oxidative process of the body is an essential element of the condition. It is only when this reaches a certain stage that the acetone bodies appear in the urine as a necessary consequence." Further, he states the acetone and aceto-acetic acid, though usually present in the urine when the coma supervenes, are not so abundant as before, but that the amount of beta-oxybutyric acid is generally much increased.

The completed history of the case, with its transient glycosuria, indicates the neurogenous origin of the glycosuria. Regarding this condition, Cammidge ${ }^{2}$ states that "such a transitory glycosuria, apparently of central origin, has been noticed in connexion with lesions of both the central and peripheral nervous system, such as tumours and hæmorrhages at the base of the brain, lesions of the floor of the fourth ventricle, cerebral and spinal meningitis, concnssion of the brain, fracture of the cervical vertebre, tetanus, and sciatica. It has also been met with after epileptic, hysteroepileptic, and apoplectic seizures, in traumatic neuroses, such as those following railway accidents, mental shocks, mental strain, worry, fatigue, and great anxiety."

The practical lesson which this case enforces is the danger of concluding that the presence of sugar in the urine of a comatose individual is necessarily due to diabetes. Further investigations and a critical survey of the history of such a case are essential for the accurate elucflation of the cause of the condition.

We have to thank Captain H. S. Morton, R.A.M.C., for the clinical history of this case, and Surgeon-General J. Dallas Edge, C.B., A.M.S., commanding the Queen Alezandra Military Hospital, for permission to publish it.

\section{THE RECURRENT TYPE OF "TRENCH" FEVER IN MESOPOTAMIA.}

BY O. F. COOMBS, M.D. LOND., M.R.C.P. LoND.,

CAPTAIN, R.A.M.C. (T.F.) ; LATELY ATTACHED TO INDIAN EXPEDITIONARY FORCE " $D$."

THE following case came under my observation recently at Amara, in Mesopotamia.

The patient, an officer aged about 36, was employed at the advanced base and had never been to the fighting line; he had been pulled down a little by one or two attacks of diarrhoea. Towards the end of April he had an attack of fever lasting two or three days, unaccompanied by rigor, and almost certainly not malarial. It seemed to belong to the category of short fevers, of which there were many examples in Amara. These attacks were generally traceable to exposure to the sun, which was beginning to get hot at that time. In this case the fever passed off, leaving the patient perhaps a little more vulnerable to the sun than before, but without definite sequelæ. Soon after a small degree of footdrop developed in the right foot, with some anæsthesia of the dorsum of the foot, but this did not seem to have any direct connexion with the febrile attack which had preceded it.

About the middle of May, after a day of premonitory malaise and depression, the temperature ran up one afternoon to $103^{\circ} \mathrm{F}$. and over. There was some mental excitement, the back and head ached, and the fall of temperature which soon followed was accompanied by a drenching sweat. After a couple of days in bed the patient returned to duty but felt very tired and slack. Just a week after this last febrile attack another one developed. The fever was not so high, but it lasted two or three days. The pains accompanying it in back and limbs were more pronounced. Four similar attacks followed at intervals of exactly six days. Each attack exhibited a little less fever and a little more prostration than those which preceded it. In the later ones the pains were more pronounced, especially in the shins and tarsal bones. These pains were much aggravated by walking, and at night they were sometimes severe enough to

1 Cammidge, P. J.: Glvcosuria and Allied Conditions, p. 214 London: Edward Arnold. 1913.

$$
\text { 2 Loc. cit., p. } 169 .
$$

prevent sleep. Latterly they tended to persist after the febrile bout was over. Possibly there was a fifth attack, but if so the rise of temperature was slight. As it occurred, if at all, during transfer from one hospltal to another, this slight rise may have been overlooked.

In all, therefore, there were six pyrexial bouts. None of them were marked by definite rigor. There was always a day of premonitory discomfort. During the two earlier apyrexial intervals the patient felt comparatively fit, but each returning attack, though in itself less severe, induced a progressive loss of strength and flesh, the latter amounting in all to nearly one quarter of the total body weight. He became very pale and a little short of breath on exertion. The pulse was persistently quickened. The bowels were constipated and the urine normal. The foot-drop already alluded to cleared up slowly with the rest in bed.

The blood was examined several times. No spirilla or other protozoa could be found. Cultures were negative and agglutination results equivocal. There was a mild polynuclear leucocytosis between the fifth and sixth attacks.

Many suggestions were offered as to the diagnosis. $\mathbf{M y}$ own view was that it might be one of the spirillar group of relapsing fevers, but this was negatived by the blood examinations, made by no less an authority than Major F. P. Mackie, I.M.S., whose researches into these diseases are well known. Captain Jamieson, R.A.M.C., recognised the similarity of the symptoms to those which had been described in Flanders under the heading of "trench fever." I was not at that time familiar with this syndrome, but since returning to England and being transferred to France $I$ have seen a number of cases of a recurrent fever which closely resembles the pyrexial illness described above.

Two other cases of a similar fever came under my notice in Mesopotamia, but I have no satisfactory notes of them. In none of the three cases was any treatment effective save evacuation from the country into a cooler climate. This corresponds with general experience of the disease : nothing does much good except rest, plenty of food, and all the other ordinary means of increasing resistance to infection.

The causation is obscure. That the climatic factor may be ruled out is proved by the occurrence of the disease in Mesopotamia, in the Balkans, in the British armies in Flanders and in the French armies in the field, and in the troops on the Volbynian front. So far as the biting insect factor is concerned, Mesopotamia is certainly one with the other war areas; except that there are added various other torments of this description to those which usually adorn the person of the soldier in the field. The whole problem is well worthy of investigation, as the disease may inflict prolonged invalidism on the soldier. Fortunately there do not appear to be any permanent sequelæ.

\section{A PRELIMINARY CONTRIBUTION ON "P.U.O. (TRENCH FEVER)."}

BY F. C. DAVIES, M.B. CAMB., M.R.C.P. LoNd.,

CAPTAIN, R.A.M.C. (T.C.) ; TEMPORARY OFFICER IN CHARGE, MEDICAI. DIVISION, NO. - GENERAL HOSPITAL ; AND

R. P. WELDON, L.R.C.P. \& S. IREL.,

CAPTAIN, R.A.M.C. (T.C.) ; PATHOLOGIS'T, NO. - GENERAT HOSPITAI.

ALMost the only real contribution to our knowledge of this disease, somewhat unfortunately termed "trench fever," which has been published, is contained in the report of the very careful and valuable work carried out under the guidance of Colonel Sir Wilmot Herringham, R.A.M.C., by Captain J. W. McNee, Captain R. J Arundel, Captain Renshaw, and Captain E. H. Brunt. Stated briefly, they have told us that this disease is almost certainly due to an organism which is probably contained in the red corpuscles of the infected persons and that the corpuscular elements of the blood alone are capable of containing and transmitting the disease. This valuable discovery is quite in accordance with what one would expect, judging by the clinical characters so markedly shown in typical cases, and which, in certain respects, are not unlike attacks of malaria. In the main. one would outline these as follows : Sudden onset occasionally with chill. Onset with indefinite but well-marked weakness and malaise, frequently indescribable so far as the patient is concerned, who, when pressed to an extremity in so many instances says, "W Well, my legs would not hold me." 